\title{
APRENDIZAJE \\ MÓVIL COMBINADO EN LA ASIGNATURA DE AICLE EN LOS GRADOS DE EDUCACIÓN PRIMARIA Y EDUCACIÓN INFANTIL
}

\section{M-LEARNING AND B-LEARNING IN THE CLIL COURSE IN THE PRIMARY AND PRE-PRIMARY EDUCATION DEGREES}

\author{
Javier Fernández Molina \\ Licenciado en Filología Inglesa, UA. \\ Máster Universitario en Formación del Profesorado, UCAM. \\ Profesor Asociado en el Departamento de Innovación y Formación Didáctica. \\ Universidad de Alicante, España. \\ E-mail: javierfmolina@ua.es ORCID: https://orcid.org/0000-0002-9273-7821

\begin{abstract}
María Felicidad Tabuenca Guevas
Licenciada en Filología Inglesa, UA.

Doctora en Investigación Educativa, UA.

Profesora Contratada Doctor en el Departamento Innovación y Formación Didáctica.

Universidad de Alicante, España.

E-mail: maria.tabuenca@gcloud.ua.es ORCID: https://orcid.org/0000-0002-7985-2614
\end{abstract}

Recepción: 21/12/2018 Aceptación: 27/12/2018 Publicación: 28/06/2019

Citación sugerida:

Fernández Molina, J. y Tabuenca Cuevas, M. F. (2019). Aprendizaje móvil y aprendizaje combinado en la asignatura de AICLE en los Grados de Educación Primaria y Educación Infantil. 3C TIC. Cuadernos de desarrollo aplicados a las TIC, 8(2), 84-101. doi: http://dx.doi.org/10.17993/3ctic.2019.82.84-101

Note: This article can be read in its original English version on:

https://www.3ciencias.com/wp-content/uploads/2019/06/3C-TIC-ED.-29_VOL.-8_No-2_art5_eng.pdf 


\section{RESUMEN}

Este trabajo presenta un estudio realizado en la Universidad de Alicante con alumnos de tercer y cuarto año del Grado de Maestro en Educación Infantil y Primaria. Conforme a la política de educación plurilingüe de la Comunidad Valenciana los estudiantes de los grados de educación pueden cursar una asignatura de Aprendizaje Integrado de Contenido y Lenguas (AICLE). En 2018 a estos estudiantes se les invitó a participar en una experiencia del uso del aprendizaje móvil y del aprendizaje combinado prestando atención al desarrollo cognitivo como parte del modelo de las 4 ces (Coyle, 2007), de la metodología AICLE. En total, dieciséis actividades basadas en tareas diseñadas por Wilden (2017), Dudley y Osvath (2016) y Kryszewska y Campbell (1992) fueron seleccionadas y adaptadas para que los estudiantes las realizaran de forma individual, en parejas y en grupos. Con el fin de promover debates y discusiones en clase, fue necesario incorporar herramientas TICS y entornos de aprendizaje virtuales. Un total de 148 alumnos, pertenecientes a tres grupos, realizaron actividades móviles y actividades combinadas dos veces por semana durante un periodo de dos meses. Los estudiantes utilizaron principalmente sus propios dispositivos, incluyendo teléfonos móviles, tabletas, ordenadores portátiles. Las actividades se centraron en tareas específicas que como futuros docentes habrán de desarrollar profesionalmente, requiriendo capacidades mentales de orden superior como crear, analizar y evaluar. El aprendizaje móvil y combinado proporcionó el marco necesario para el desarrollo de las actividades y objetivos de la experiencia.

\section{PALABRAS CLAVE}

AICLE, Aprendizaje Móvil, Aprendizaje Combinado, TICs, Formación del Profesorado. 
Ed. 29 Vol. 8 N. 2 Junio - Septiembre 2019 DOI: http://dx.doi.org/10.17993/3ctic.2019.82.84-101

\section{ABSTRACT}

This paper presents a study carried out at the University of Alicante with third and fourth year students training to be future primary and pre-primary teachers. The Valencian Community has a plurilingual education policy; therefore, students on the education degrees can do a course on Content and Language Integrated Learning (CLIL). In 2018, these students were invited to participate on an experience using mobile learning and blended learning to focus on the cognitive development in CLIL as part of the 4-C wheel model (Coyle, 2007). Consequently, sixteen activities based on tasks previously designed by Wilden (2017), Dudley and Osvath (2016) and Kryszewska and Campbell (1992) were selected and adapted for the students to carry them out individually, in pairs, or in groups. To this end, additional ICT tools and virtual learning environments (VLES) were incorporated to promote debates and discussion in class. In total 148 students, belonging to three groups, did M-learning and B-learning activities twice a week during a two-month period. Students primarily used their own devices, which included mobile phones, tablets and laptops. The activities focused on specific tasks future teachers will have to carry out professionally and required high order thinking skills such as creating, analyzing and evaluating. The overall results illustrate how M-learning and B-learning did provide the necessary framework for the development of the activities and objectives of the experience.

\section{KEYWORDS}

CLIL, M-learning, B-learning, ICTs, Teacher training. 


\section{INTRODUCCIÓN}

La incorporación del Marco Común Europeo de Referencia para las lenguas (Consejo de Europa, 2001) a la realidad lingüística de la Comunidad Valenciana, donde existe una lengua cooficial, ha conducido a una aproximación plurilingüe (Cenoz y Gorter 2013; Cummins, 2007; Esteve, et al., 2015; García y Sylvan, 2011) en el currículo en los últimos años. La realidad del aula incluye la coexistencia y uso de las dos lenguas oficiales, así como la lengua extranjera. Según García y Silvan (2001), "debemos aprender a enseñar individuos dentro de una clase multilingüe" (p. 386). En aras a alcanzar el éxito en la enseñanza de una lengua adicional, en este caso el inglés, el recurso que más ayuda es el Aprendizaje Integrado de Contenido y Lengua Extranjera (AICLE), con el que una o más asignaturas o partes de una asignatura son enseñadas por medio de una lengua distinta a la de los estudiantes (Coyle, 2007; Marsh, et al., 2012).

Como parte de la formación didáctica en los Grados de Educación en la Universidad de Alicante los estudiantes pueden cursar una asignatura de AICLE. En esta asignatura, no solo se define la noción de AICLE y se presentan sus características más relevantes, sino que adicionalmente se guía a los estudiantes en la metodología, pedagogía y prácticas AICLE. Una vez se gradúen, ellos impartirán docencia tanto a alumnos de infantil como de primaria y, por tanto, es de vital importancia que adquieran de forma correcta las bases de este nuevo paradigma educativo. El objetivo de este estudio, con nuestros estudiantes de los grados de educación, fue trabajar con el modelo de la rueda 4 Cs (Coyle, 2002), más específicamente en lo referido a la cognición, puesto que 'AICLE debería suponer un reto cognitivo para los estudiantes, independientemente de su habilidad. El aprendizaje AICLE proporciona un escenario rico para desarrollar habilidades cognitivas en conjunción con ambas, la lengua de uso general (basic interpersonal communication skills) y la lengua académico-cognitiva (cognitive-academic language proficiency)", (p. 28). La importancia del desarrollo e implicación cognitiva es un tema determinante identificado por muchos estudios como los de Hakuta, Ferdman y Díaz (1986), Puchta y Williams (2011), y Shakkour (2014), entre otros. La intención era que los estudiantes adquirieran una comprensión más profunda en el dominio cognitivo relativo al pensamiento crítico y creativo, en relación a las habilidades cognitivas de orden superior (higher order thinking skills) en contraposición a las habilidades cognitivas de orden inferior (lower order thinking skills) según la Taxonomía de Krathwohl (2002) como revisión de la Taxonomía de Bloom. 
Según detalló Meyer (2010), una planificación exitosa junto con estrategias didácticas, son de importancia vital cuando se intenta lograr sesiones AICLE de calidad. Es necesario concienciar a los futuros docentes que la adquisición del lenguaje supone no solo la manipulación deliberada del lenguaje para mejorar el aprendizaje sino también ser un mejor oyente, polifacético y menos distraído. Para este fin, es necesario trabajar en la visualización, asociación, en el uso de pistas en el proceso lector-comprensor, y reglas nemotécnicas para lograr tener estudiantes con mejores habilidades para la resolución de problemas y que sean más creativos. Esto requiere de una importante entrada de información (input), de técnicas de andamiaje, junto con una interacción rica y una producción incentivada, lo que ha supuesto nuestro marco de partida. Meyer (2010) considera las HOTS como la clave del éxito en la era de la información. Por esta razón, nuestra intención era proporcionar actividades que abarcaran estos elementos.

Es necesario trabajar en la visualización, asociación, en el uso de pistas en el proceso lector-comprensor, y reglas nemotécnicas para lograr tener estudiantes con mejores habilidades para la resolución de problemas y que sean más creativos.

A los estudiantes se les solicitó que participaran en actividades usando supuestos de aprendizaje móvil y aprendizaje combinado, puesto que ambos pueden proporcionar retos cognitivos (Huffman y Hahn, 2015). Algunos de los motivos adicionales para elegir tareas que requirieran el uso de aprendizaje móvil y el aprendizaje combinado en nuestra investigación se pueden encontrar en la introducción de Wilden (2017). El autor enfatiza lo difícil que resulta a los docentes interrumpir la adicción de nuestros estudiantes a comprobar constantemente sus dispositivos, a mantenerse conectados casi al minuto en sus redes sociales, incluso a únicamente visionar de forma rutinaria y sin prestar atención un número sin fin de vídeos, así como material similar. Es un hecho que vivimos en un mundo móvil, donde la tendencia es a estar más y más conectados y, por tanto, hay muy poco que podamos hacer los docentes para parar esta tendencia. Por lo tanto, él cuestiona si no sería más productivo aprovecharse de estos recursos que tenemos a nuestra disposición, que de hecho no encuentra resistencia por parte de los estudiantes, para nuestros propósitos didácticos diarios.

Adicionalmente, lo que fue de interés para nuestro propósito de Dudley y Osvath (2017) y Kryszewska y Campbell (1992) fue su aproximación a los retos actuales en materia educativa desde su particular 
perspectiva dentro del ámbito AICLE. Fue nuestra intención que las actividades lograran incentivar una comunicación efectiva en el aula, sobreponiéndonos al desequilibrio entre el dominio del idioma y su habilidad para desarrollar las tareas designadas (Dudley y Osvath, 2017). Es un problema conocido que con demasiada frecuencia la temática habitual en las clases de inglés como lengua extranjera están, o bien más allá de la experiencia vital de los estudiantes, o simplemente son un fracaso en el aula. En muchas actividades nuestros alumnos tuvieron que compartir sus resultados con sus compañeras y compañeros de clase una vez que habían cumplido con su aprendizaje autónomo o con el aprendizaje basado en una tarea (Carless, 2002; Ellis, 2003). Adicionalmente, con cada presentación realizada, al resto del alumnado se les solicitó que proporcionaran una retroalimentación en forma de evaluación entre pares. Las actividades fueron incorporadas a la dinámica del curso en la forma de actividades de preparación de la sesión, como continuación de la misma o para fomentar futuro debate y disertación.

\section{METODOLOGÍA}

Las actividades que realizaron los estudiantes fueron seleccionadas de entre las obras y seminarios de los autores Wilden (2017), Dudley y Osvath (2017) Kryszewska y Campbell (1992) y Hird (2018). A continuación, proporcionaremos la descripción del contexto y de los participantes, junto con la descripción de las actividades desarrolladas, así como la actuación de los estudiantes. El interés en una aproximación en el marco AICLE por parte de Kryszewska y Campbell (1992), en contraposición con el inglés como medio de instrucción (EMI), se reflejó en nuestro estudio por medio del principio de evitar la tiranía del correcto o equivocado. Según los autores, una equivocación es un regalo para la clase, puesto que supone el punto de partida de la discusión y el debate. Las actividades que recomiendan, algunas de las cuales desarrollamos en nuestra investigación, no tienen una única respuesta correcta. Los autores ciertamente consideran que son las personas que tienen soluciones alternativas, opciones, las que empujan el mundo hacia delante. Esta concepción se le transmitió al alumnado que formó parte de este estudio. 


\subsection{DESCRIPCIÓN DEL CONTEXTOY DE LOS PARTICIPANTES}

148 estudiantes pertenecientes al tercer y cuarto curso de los grados de educación infantil y educación primaria fueron seleccionados para participar en esta experiencia. A pesar de que la asignatura AICLE es de carácter opcional en el plan de estudios, el aprobar el curso les permite parcialmente cumplir con el requisito lingüístico mínimo para obtener la acreditación de idioma extranjero (B1 según el Marco Común Europeo de Referencia para las lenguas) necesaria para graduarse. Debido a esto, los estudiantes que tomaron parte en este estudio no son solo aquellos que serán especialistas en inglés dentro de la educación infantil y primaria, sino también futuros profesores de otras especialidades por lo que, nos encontramos con diferencias sustanciales de competencia lingüística en inglés por parte del alumnado.

La asignatura AICLE se imparte de forma intensiva en los primeros dos meses del curso académico, como resultado, hay cuatro sesiones por semana de dos horas cada una, lo que permite llevar a cabo un trabajo intensivo. La asignatura abarca tanto teoría como práctica, y las actividades de aprendizaje móvil y combinado propuestas fueron parte de las tareas requeridas en la asignatura.

La asignatura AICLE se imparte de forma intensiva en los primeros dos meses del curso académico, como resultado, hay cuatro sesiones por semana de dos horas cada una, lo que permite llevar a cabo un trabajo intensivo.

\subsection{DESCRIPCIÓN DE LOS AGRUPAMIENTOS Y DE LOS PARTICIPANTES}

Las actividades serán descritas en el mismo orden que fueron desarrolladas a lo largo del curso. Algunas actividades podían hacerse de forma individualizada, otras en parejas y un tercer grupo por medio de trabajo en grupo. A continuación, describiremos las actividades que se solicitó a los estudiantes que se realizaran, y brevemente como se llevaron a cabo.

Para comenzar, se comentarán las actividades realizadas por parejas. La actividad introductoria consistía en seleccionar una foto de entre las guardadas en sus dispositivos, entonces los estudiantes tenían que realizar tres tareas. La primera consistía en presentarse a ellos mismos a aquellas compañeras y compañeros del curso que no conocieran de cursos anteriores, haciendo uso de la 
foto. A continuación, tenían que encontrar un compañero para, sin enseñar su propia foto y por medio de interacción oral en inglés, hacer preguntas para averiguar en qué consistía la foto de la otra persona. Una vez se hubiera alcanzado un consenso sobre el aspecto que debía tener la foto del otro estudiante, entonces se les permitía mostrar su propia foto. Por último, debían comparar y contrastar ambas fotos (Tabla 1).

Tabla 1. Actividades de aprendizaje móvil en pareja de preparación.

\begin{tabular}{|c|c|}
\hline \multicolumn{2}{|c|}{ AICLE - APRENDIZAJE MÓVIL TRABAJO EN PAREJA DE PREPARACIÓN } \\
\hline \multirow{4}{*}{$\begin{array}{l}\text { Selecciona una } \\
\text { fotografía de tu } \\
\text { teléfono móvil }\end{array}$} & Utilízala para presentarte a ti mismo. \\
\hline & $\begin{array}{l}\text { Haz preguntas: intenta averiguar de qué va la foto de } \\
\text { vuestra/o compañera/o... }\end{array}$ \\
\hline & $\begin{array}{ll}- & \text { ¿Hay gente? } \\
\text { - } & \text { ¿Es un lugar? } \\
\text { - ¿Qué está pasando? }\end{array}$ \\
\hline & $\begin{array}{l}\text { Compara y contrasta } \\
\text { - } \quad \text { En mi foto hay más... } \\
\text { - } \quad \text { Mi foto no es tan... como... }\end{array}$ \\
\hline
\end{tabular}

Fuente: elaboración propia.

En el segundo grupo de actividades, se solicitó al estudiantado que trabajaran de forma individual. Adicionalmente, en el caso de la segunda y tercera tarea, debieron preparar una presentación para el resto de la clase. En cuanto a la audiencia, se les solicitó que proporcionaran retroalimentación e información de recopilación.

Tabla 2. Actividades individuales de aprendizaje móvil de preparación.

\begin{tabular}{|c|c|}
\hline $\begin{array}{c}\text { AICLE - APRENDIZAJE MÓVIL ACTIVIDADES INDIVIDUALES DE PREPARACIÓN } \\
\text { Fotos de phrasal } \\
\text { verbs }\end{array}$ & Muestra tus fotos de phrasal verbs vivientes. Competición. \\
\hline $\begin{array}{c}\text { Actividad de alarma } \\
\text { y selfies }\end{array}$ & $\begin{array}{c}\text { Programa la alarma a cinco horas aleatorias y toma un selfie cada } \\
\text { vez que suene la alarma. Crea un collage, un power point o un video } \\
\text { y explica qué estabas haciendo cuando se tomaron los selfies. }\end{array}$ \\
\hline $\begin{array}{c}\text { Actividad del grafiti } \\
\text { Emoji para el } \\
\text { profesor }\end{array}$ & $\begin{array}{c}\text { Envía un emoji al profesor indicando cuáles son tus sentimientos } \\
\text { hacia la asignatura. Añade comentarios. }\end{array}$ \\
\hline
\end{tabular}

Fuente: elaboración propia. 
En lo que se refiere a la primera actividad, se solicitó a los estudiantes de la asignatura de AICLE que hicieran cuatro fotos de phrasal verbs en acción y fuera del aula como preparación para la sesión del día siguiente. Por ejemplo, un dedo y un enchufe podían significar turn on o turn off, sus zapatillas y un dedo en el cordón a su vez podía significar tie up o tie down, etc. Una vez en clase, fuimos al pasillo de la facultad y organizando dos líneas paralelas, tenían que competir con los compañeros situados frente a cada una/o averiguando los phrasal verbs de aquellos. La persona que averiguaba correctamente se movía hacia delante en la línea y el que perdía hacia atrás. Así es como se desarrolló la competición, teniendo lugar al comienzo de la sesión. La cognición como parte del aprendizaje de una lengua (Coyle, 2007) emergió de forma recurrente.

La segunda actividad consistía en, al menos teóricamente, que las y los alumnos tomaran cinco selfies durante la semana, uno por día laboral, a diferentes horas. Primero, se les pidió que programaran la alarma de su móvil para posteriormente darles las indicaciones sobre qué tenían que hacer. Aunque se asumía un cierto grado de trampa y de hecho así sucedió, tal como algún estudiante llevando siempre la misma ropa en todas las fotos, los resultados fueron excepcionales. Todo el alumnado disfrutó mucho hablando sobre ellos mismos, aun cuando la actividad se desarrolló en inglés.

En cuanto a la actividad del grafiti, básicamente se les solicitó que bien eligieran un grafiti que conocieran de sus ciudades o pueblos de residencia, o grafitis famosos que se hubieran encontrado en Londres o en cualquier otro lugar. La única exigencia fue que debían grabarse en video o mediante un audio comentando los sentimientos que les inspiraba aquel grafiti.

Por último, a estas alturas del curso, se solicitó a las y los estudiantes que enviaran un emoji al profesor que mostrara cómo se sentían hasta ese momento en el curso de AICLE. Para ello, se había creado con carácter previo un grupo de WhatsApp que facilitara la comunicación en el grupo.

Tabla 3. Actividades de aprendizaje móvil en parejas.

\begin{tabular}{|c|c|}
\hline \multicolumn{2}{|r|}{ AICLE - APRENDIZAJE MÓVIL TRABAJO EN PAREJA } \\
\hline Pic Collage & $\begin{array}{c}\text { Crea un pic collage con seis fotos seleccionadas de tu dispositivo y } \\
\text { preséntate a ti mismo a gente desconocida. }\end{array}$ \\
\hline Pic Collage & $\begin{array}{l}\text { En un nuevo pic collage, cinco fotos tienen que estar relacionadas de } \\
\text { alguna manera. Intenta averiguar la que no encaja en las seis fotos de } \\
\text { tu compañera/o. }\end{array}$ \\
\hline
\end{tabular}




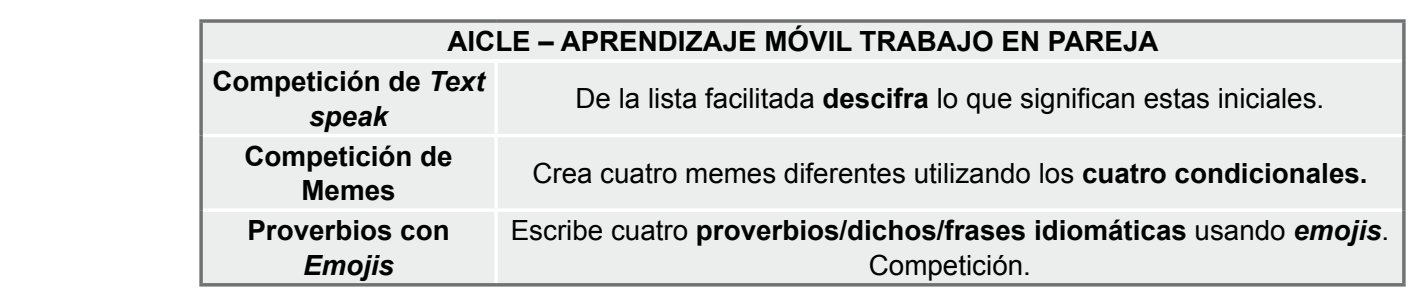

Fuente: elaboración propia.

En relación a las actividades a ser desarrolladas en parejas (Tabla 3), las dos primeras requerían que conocieran alguna aplicación que les permitiera crear un collage, a quienes no conocían ninguna se les facilitó la app pic collage creator. Una vez se hubieron descargado la herramienta, para la primera actividad se les solicitó que se presentaran ellas y ellos mismos a aquellas compañeras/os que aún no conocieran, para que nuevamente salieran de su zona de confort. Por medio de los pic collage con fotos elegidas por sí mismos se consiguió que fueran más habladores.

En cuanto a la segunda actividad con el pic collage, se introdujo un grado de dificultad puesto que cinco de las fotos en el collage debían estar relacionadas de alguna manera y solo una debía ser de naturaleza diferente. La actividad consistía en averiguar cuál era la imagen que no encajaba y porqué.

Para la tercera tarea, a los estudiantes se les facilitó una serie de palabras que son de hecho frecuentemente usadas en los textos escritos en los móviles por gente joven angloparlante como argot. El objetivo era que intentaran descifrar el significado del mayor número de ellas. Esta actividad se basa en una similar de un seminario de Hird (2018) y fue adaptada.

La competición de memes, que fue recibida con entusiasmo por parte de los tres grupos de la asignatura AICLE fue simple. En parejas, tenían que crear cuatro memes con el único requisito de usar todos y cada uno de los cuatro condicionales del inglés. Periódicamente, al inicio de cada sesión se les ofreció con carácter voluntario presentar al resto de la clase sus propias creaciones. La temática fue ilimitada y la única frontera o límite que se estableció fue la norma conocida como "la abuela en clase". Este concepto hace referencia al hecho de que, si la abuela de la alumna/del alumno estuviera en clase, si estimaría correcto o no lo que se presenta, lo que se dice. Es decir, si tu abuela estuviera en clase ¿presentarías de igual manera tus memes? 
Por último, la competición de proverbios, dichos y frases idiomáticas requiere una mención distintiva. La sesión estaba diseñada para ofrecer un entorno de aprendizaje remoto. El objetivo era comprobar que una carga cognitiva adecuada (Choi, Van Merriënboer y Paas, 2014) se podía lograr en un entorno diferente al del aula. A cada grupo se le indicó que los primeros veinte minutos de la siguiente sesión tendría lugar en la cantina de la facultad. Ellas y ellos debían distribuirse de forma aleatoria por la cantina en parejas con sus teléfonos móviles encendidos y sus baterías cargadas. En ese momento no se les facilitó ninguna instrucción adicional. Al día siguiente, a la hora convenida, se les informó que nuevamente se trataba de escribir en el grupo de WhatsApp proverbios o dichos o frases idiomáticas en inglés haciendo uso de los emojis. Es decir, una pareja escribiría los emojis y el resto debería adivinar qué se había escrito. Al principio, les llevó un poco de tiempo entrar en la dinámica de la actividad, pero pronto todos comenzaron a participar muy activamente. Terminó siendo una actividad muy dinámica que duró más de lo previsto inicialmente.

Tabla 4. Actividades de aprendizaje móvil en grupos.

\begin{tabular}{|c|c|}
\hline \multicolumn{2}{|r|}{ AICLE - APRENDIZAJE MÓVIL EN GRUPOS } \\
\hline Competición de Rebus & Descifra lo que cada uno de estos Rebuses significa. \\
\hline Historia colaborativa con móvil & $\begin{array}{c}\text { Cada miembro del grupo escribe una frase en un dispositivo común (cinco o } \\
\text { seis palabras) y lo pasa a la siguiente persona. }\end{array}$ \\
\hline Diseña un emoji & $\begin{array}{l}\text { Acordad un significado a la lista de emojis que se facilita. A continuación, crea } \\
\text { un emoji para este curso AICLE. }\end{array}$ \\
\hline $\begin{array}{c}\text { Diseña un anuncio para este } \\
\text { curso }\end{array}$ & $\begin{array}{c}\text { Usando los tiempos futuros y un dispositivo, diseñad un anuncio para este } \\
\text { curso. }\end{array}$ \\
\hline
\end{tabular}

Fuente: elaboración propia.

Las cuatro actividades en la Tabla 4 fueron diseñadas para que se realizaran en grupos, comenzando por una competición de Rebus. Utilizando un generador de rebuses online, varias frases fueron facilitadas a los alumnos para que trabajaran con ellas en grupos. Únicamente debían descubrir cuál era la frase oculta. Resultó ser una actividad que supuso un desafío que sorprendentemente desarrolló en ellos los mayores instintos competitivos.

Otra actividad que se pudo desarrollar de manera sencilla fue la historia colaborativa. En esta ocasión, debían hacer uso de uno de los móviles de algún miembro del grupo. Cada persona escribiría cinco o seis palabras y pasaría el móvil a la siguiente persona hasta que la historia se completara. Al final de la sesión se leyeron en voz alta todas las historias que los distintos grupos habían creado. 
Las últimas dos actividades de este grupo requirieron de una presentación posterior frente al resto de la clase. La tercera actividad consistió en diseñar un nuevo emoji que describiera de la forma más fidedigna el curso, mientras que la cuarta supuso diseñar un anuncio del curso para los alumnos del siguiente año académico. Se les solicitó que utilizaran tiempos futuros, así como herramientas electrónicas. Ambas actividades supusieron el uso de habilidades cognitivas de orden superior tales como crear, evaluar y analizar.

Tabla 5. Actividades de aprendizaje combinado en grupos.

\begin{tabular}{|c|c|}
\hline $\begin{array}{c}\text { Describid un cuadro } \\
\text { Escribid cinco preguntas sobre el cuadro que empiecen por ¿por qué? } \\
\text { Responded dichas preguntas. }\end{array}$ \\
\hline $\begin{array}{c}\text { Alfabeto de medios } \\
\text { de transporte }\end{array}$ & $\begin{array}{c}\text { En grupos, nombrad divertidos, creativas, existentes medios de } \\
\text { transporte, tanto ficticios como reales. }\end{array}$ \\
\hline
\end{tabular}

Fuente: elaboración propia.

El aprendizaje combinado (Tabla 5) se centró en dos tareas basadas en actividades desarrolladas por Kryszewska y Campbell (1992). En cuanto a la primera, los estudiantes debían agruparse en grupos pequeños, sobre la pantalla se proyectaría un cuadro y ellas y ellos debían preguntar cinco cuestiones sobre el cuadro. Estas preguntas debían comenzar por ¿por qué?, entonces, hubo una sesión recapitulativa para compartir las preguntas con el resto de grupos. Esta sesión les permitió obtener una idea más profunda sobre el cuadro, llegar más a los detalles, según lo sugerido por Howard Gardner cuando se refiere a la alfabetización audiovisual.

La segunda de las actividades supuso un menor esfuerzo, si bien todavía requería de habilidades cognitivas de orden superior por parte de las y los alumnos en cuanto a juzgar y realizar hipótesis, mientras se combinaban con habilidades cognitivas de orden inferior como listar, clasificar y organizar. Debían proporcionar existentes, divertidos y creativos medios de transporte cuya primera letra siguiera el orden alfabético, radicando ahí su dificultad. Esta actividad se puede adaptar a cualquier temática dentro de la clase de inglés como lengua extranjera.

A lo largo de esta experiencia, los estudiantes subieron a una plataforma virtual de aprendizaje en formato de portfolio digital el compendio de actividades desarrolladas en este curso. Según Paulson, Paulson y Meyer (1991) un portfolio permite a los alumnos revelar mucho sobre sí 
mismos convirtiéndose el portfolio en una ventana a las mentes del alumnado. Si bien, se incidió particularmente en la producción durante las clases, principalmente de forma oral durante el desarrollo de estas actividades, no era nuestra intención únicamente evaluar el desempeño de nuestros alumnos sino promover y aumentar habilidades cognitivas de orden superior. Por lo tanto, la creación del portfolio digital fue necesario para este propósito.

\section{CONCLUSIONES}

El principal objetivo de esta experiencia fue concienciar a los futuros profesores de primaria e infantil de que el aprendizaje móvil como el aprendizaje combinado puede llevar a un uso de habilidades cognitivas de orden superior mientras se incrementa el conocimiento de los estudiantes de una lengua extranjera in un entorno de una clase multicultural. Los estudiantes deben tener en cuenta que existen otras formas de enseñar inglés (siguiendo la metodología AICLE) y que la cognición es esencial en el aprendizaje de lenguas por parte de los estudiantes. Cuando ellos, en un futuro, enseñen a estudiantes de infantil y sobre todo de primaria, podrán utilizar dispositivos electrónicos para un aprendizaje móvil, así como muchos otros recursos de aprendizaje combinado en sus clases en aras a incrementar las habilidades cognitivas de orden superior tales como crear, analizar y evaluar para obtener los más óptimos resultados posibles. Adicionalmente, queremos destacar que con el grupo de estudiantes con el que se trabajó, el aprendizaje móvil junto con el aprendizaje combinado condujo a un incremento del entusiasmo de los estudiantes, de su autoestima y de la cohesión del grupo. Quizás esto se deba al hecho de que se incentivaron durante el desarrollo de las actividades el translenguaje (García y Sylvan, 2011) y dinámicas positivas de grupo (Clément, Dornyei y Noels, 1994; Dornyei, 2003) sin embargo, esto necesita ser estudiado más en profundidad.

El grupo de estudiantes con el que se trabajó, el aprendizaje móvil junto con el aprendizaje combinado condujo a un incremento del entusiasmo de los estudiantes, de su autoestima y de la cohesión del grupo. 


\section{REFERENCIAS BIBLIOGRÁFICAS}

Carless, D. (2002). Implementing task-based learning with young learners. ELT journal, 56(4), 389396.

Cenoz, J., y Gorter, D. (2013). Towards a plurilingual approach in English language teaching: Softening the boundaries between languages. Tesol Quarterly, 47(3), 591-599.

Choi, H. H., Van Merriënboer, J. J., y Paas, F. (2014). Effects of the physical environment on cognitive load and learning: towards a new model of cognitive load. Educational Psychology Review, 26(2), 225-244.

Council of Europe. (2001). Common European Framework of Reference for Languages: learning, teaching, assessment. Cambridge: Cambridge University Press.

Coyle, D. (2002). Relevance of CLIL to the European Commission's Language Learning Objectives. In D. Marsh, CLIL/EMILE The European Dimension, 27-28. Jyväskylä: UniCOM.

Coyle, D. (2007). Content and language integrated learning: Towards a connected research agenda for CLIL pedagogies. International journal of bilingual education and bilingualism, 10(5), 543-562.

Glément, R., Dörnyei, Z., y Noels, K. A. (1994). Motivation, self-confidence, and group cohesion in the foreign language classroom. Language learning, 44(3), 417-448.

Cummins, J. (2007). Rethinking monolingual instructional strategies in multilingual classrooms. Canadian Fournal of Applied Linguistics, 10(2), 221-240.

Dörnyei, Z. (2003). Attitudes, Orientations, and Motivations in Language Learning: Advances in Theory, Research, and Applications. Language Learning, 53(1), 3-32.

Dudley, E., y Osváth, E. (2016). Mixed Ability Teaching-Into the Classroom. Oxford: Oxford University Press.

Ellis, R. (2003). Task-based language learning and teaching. Oxford: Oxford University Press. 
Esteve, O., Fernandez, F., Martin-Peris, E., y Atienza, E. (2017). The Integrated Plurilingual Approach: A didactic model providing guidance to Spanish schools for reconceptualizing the teaching of additional languages. Language and Sociocultural Theory, 4(1), 1-24.

García, O. y Sylvan, G. E. (2011). Pedagogies and practices in multilingual classrooms: Singularities in pluralities. The Modern Language Fournal, 95(3), 385-400.

Hakuta, K., Ferdman, B. M. y Diaz, R. M. (1986). Bilingualism and cognitive development: Three perspectives and methodological implications. UCLA: Center for Language Education and Research.

Hird, J. (2018). Aspects of Contemporary English: Teaching a changing language. Oxford University.

Huffman, W. B., y Hahn, S. (2015). Cognitive Principles in Mobile Learning Applications. Psychology, 456-463

Krathwohl, D. R. (2002). A revision of Bloom's taxonomy: An overview. Theory into practice, 41(4), 212-218.

Kryszewska, G. G. H., y Campbell, G. (1992). Learner-based teaching. Oxford: Oxford University Press.

Marsh, D., Mehisto, P., Wolff, D., y Frigols Martin, M. J. (2012). European framework for CLIL teacher education. Graz: European Centre for Modern Languages.

Meyer, O. (2010). Towards quality CLIL: successful planning and teaching strategies. PULSO. Revista de Educación, (33), 11-29.

Paulson, F. L., Paulson, P. R., y Meyer, G. A. (1991). What makes a portfolio a portfolio? Educational leadership, 48(5), 60-63.

Puchta, H. y Williams, M. (2011). Teaching Young Learners to Think. Innsbruck and Cambridge: Helbling Languages and Cambridge University Press. 
Shakkour, W. (2014). Cognitive Skill Transfer in English Reading Acquisition: Alphabetic and Logographic Languages Compared. Open Journal of Modern Linguistics, 4, 544-562.

Wilden, S. (2017). Mobile Learning. Oxford: Oxford University Press. 
Ed. 29 Vol. 8 N.o. 2 Junio- Septiembre 2019
Dol: httpi///dxdoio.org/10.17993/3ctic.2019.82.84-101

101 